\title{
Gray-Value Based Registration of CT and MR Images by Maximization of Local Correlation
}

\author{
J. Weese ${ }^{1}$, P. Rösch ${ }^{1}$, T. Netsch ${ }^{1}$, T. Blaffert ${ }^{1}$, and M. Quist ${ }^{2}$ \\ 1 Philips Research Laboratories, Division Technical Systems \\ Röntgenstraße 24-26, D-22335 Hamburg, Germany \\ 2 EVM Advanced Development, Philips Medical Systems Nederland B.V. \\ Veenpluis 4-6, NL-5680 DA Best, The Netherlands
}

\begin{abstract}
For gray-value based multi-modality registration the similarity measure is essential. Excellent results have been obtained with mutual information for various modality combinations. In this contribution we consider local correlation as similarity measure for multi-modality registration. Using a software phantom it is analyzed why local correlation is suitable for this registration task whereas direct gray-value correlation itself is usually not. It is shown that registration with local correlation can be done using only a fraction of the image volume offering an opportunity to accelerate the algorithm. Within validation, registration of the phantom images, two simultaneously acquired dual contrast MR images, and a clinical CT-MR data set has been studied. For comparison, the data sets have also been registered with mutual information. The results show that not only mutual information, but also local correlation is suitable for gray-value based multi-modality registration.
\end{abstract}

\section{Introduction}

There are several clinical applications for which images from different modalities are required, because they provide complementary information. A typical example is the use of MR and CT images for radiation therapy planning. Another application is the combination of functional images with the underlying anatomy represented e.g. by an MR image. Different patient posture and positioning during image acquisition complicate the interpretation of the images, and various multi-modality registration algorithms have been developed therefore [1]

For gray-value based methods the similarity measure is essential. Correlation, for instance, relies on a linear relationship between the gray-values of the images to be registered. Such a linear relation cannot be expected for multi-modality data sets and gray-value correlation cannot be applied directly, therefore. To make correlation or the correlation coefficient suitable for CT-MR registration, several pre-processing techniques have been proposed. Studholme et al. [2] applied a non-linear remapping of the CT intensities. Maintz et al. [3] computed feature images with edges or ridges. Mutual information is much more suited for multi-modality registration, because it does not rely on a functional relationship between the gray-values. This distinguishes mutual information from other 
similarity measures. Multi-modality registration by maximization of mutual information has been proven to be very successful and excellent results have been obtained for various modality combinations (CT-MR, MR-PET etc.) $[2,4,5]$.

In this contribution we consider the registration of CT and MR images by maximization of local correlation [6]. To compute local correlation, for each pixel in the reference image a neighborhood is defined, the correlation coefficient between this neighborhood and the corresponding neighborhood in the image to be registered is evaluated, and all resulting correlation coefficients are summed up. At the first glance, it is not obvious, why local correlation is suitable for multi-modality registration. In order to answer this question, a multi-modality data set has been simulated. Using this software phantom, the differences between correlation and local correlation are thoroughly discussed in the following section. In section 3 the experiments are described, which have been performed for validation. Apart from the software phantom, two simultaneously acquired MR images and a clinical CT-MR data set have been used. For comparison, the validation experiments have also been carried out using mutual information. The results are discussed in section 4 . Section 5 contains the conclusions.

\section{Local Correlation and Multi-Modality Registration}

The starting point for the discussion of correlation and local correlation is a software phantom representing two images of different modality. This phantom is introduced in the first subsection. On the basis of this phantom the properties of correlation and local correlation are discussed in the second and third subsection.

\subsection{Software Phantom}

The software phantom represents a simple model of a multi-modality image set. It is based on the assumption that each type of tissue leads to a characteristic gray-value which depends on the physical effect used for imaging and the material parameters of the tissue. These gray-values are therefore different for different modalities and there is in general no functional relationship between the grayvalues of multi-modality images.

The software phantom was generated with a segmentation editor based on the watershed algorithm of Vincent and Soille [7]. To enable interactive editing, markers can be placed which are handled as constraints within region merging. With this editor an MR image has been segmented into six different structures (skin, ventricles, eyes, cortex, background, rest), and two images of different modality have been simulated by assigning random gray-values to the segmented structures. The gray-values are listed in Tab. 1. A slice of each of the simulated multi-modality images is shown in Fig. 1. 
Table 1. Gray-values assigned to the structures of the segmented MR image.

\begin{tabular}{l|c|c|c|c|c|c|} 
& skin & ventricles & cortex & eyes & background & rest \\
\hline Image a & 48 & 62 & 70 & 123 & 0 & 5 \\
Image b & 15 & 83 & 31 & 101 & 0 & 53 \\
\hline
\end{tabular}
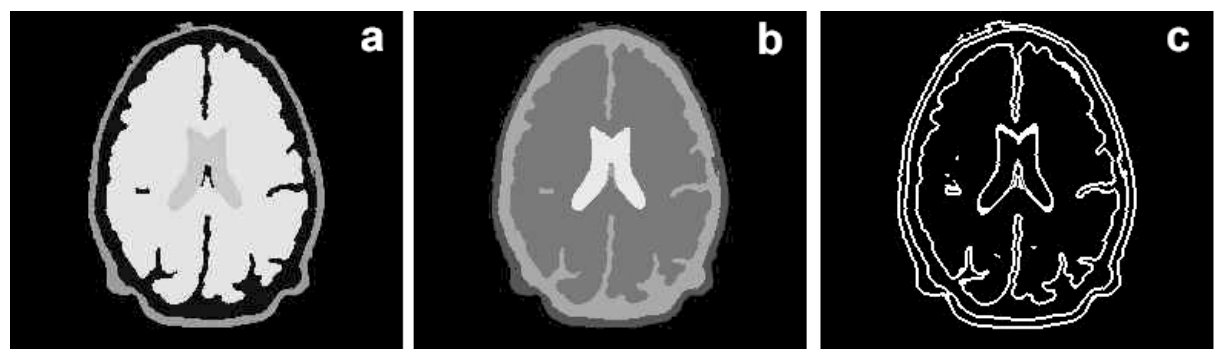

Fig. 1. Simulated multi-modality images $(\mathrm{a}, \mathrm{b})$ and spatial distribution of the contributions to the local correlation for $r=1$ (c).

\subsection{Correlation}

A possibility to register two images of the same modality is the minimization of the mean-square gray-value difference

$$
D(\boldsymbol{\omega}, \boldsymbol{t})=\frac{1}{\# M} \sum_{\boldsymbol{i} \in M}\left(I_{1}(\boldsymbol{i})-I_{2}(\boldsymbol{i})\right)^{2}, \quad \boldsymbol{i}=\left(i_{x}, i_{y}, i_{z}\right)
$$

with respect to the translation $\boldsymbol{t}$ and the rotation $\boldsymbol{\omega}$ of a rigid transformation. In this equation, $I_{1}(\boldsymbol{i})$ denotes the reference image. The image $I_{2}(\boldsymbol{i})$ is registered and depends on the transformation parameters $\boldsymbol{t}$ and $\boldsymbol{\omega}$. The summation is performed over all pixels in the overlap region given by the set $M$.

This approach can be considered as a least-squares method. If the gray-values of the images differ by a gray-value scaling $I_{0}$ and a gray-value offset $\Delta I$, the concept of least-squares methods suggests to insert these parameters into (1) and define the registration result by the minimum of

$$
D^{\prime}(\boldsymbol{\omega}, \boldsymbol{t})=\frac{1}{\# M} \sum_{\boldsymbol{i} \in M}\left(I_{1}(\boldsymbol{i})-I_{0} I_{2}(\boldsymbol{i})-\Delta I\right)^{2}
$$

with respect to the transformation parameters $\boldsymbol{t}$ and $\boldsymbol{\omega}$, the scaling $I_{0}$, and the offset $\Delta I$. Minimization with respect to the latter two parameters leads to

$$
D^{\prime}(\boldsymbol{\omega}, \boldsymbol{t})=\frac{\sum_{\boldsymbol{i} \in M}\left(I_{1}(\boldsymbol{i})-\bar{I}_{1}\right)^{2}}{\# M}-\frac{\left(\sum_{\boldsymbol{i} \in M}\left(I_{1}(\boldsymbol{i})-\bar{I}_{1}\right)\left(I_{2}(\boldsymbol{i})-\bar{I}_{2}\right)\right)^{2}}{\# M \sum_{\boldsymbol{i} \in M}\left(I_{2}(\boldsymbol{i})-\bar{I}_{2}\right)^{2}}
$$


with $\bar{I}_{1}$ and $\bar{I}_{2}$ denoting the average gray-values in the overlap region for the reference image and the image to be registered, respectively. The first term in this equation is the gray-value variance of the reference image $I_{1}(\boldsymbol{i})$ within the overlap region. The second term represents the reduction of the gray-value variance due to adaptation of the image $I_{2}(\boldsymbol{i})$. The ratio of both terms

$$
C^{2}(\boldsymbol{\omega}, \boldsymbol{t})=\frac{\left(\sum_{\boldsymbol{i} \in M}\left(I_{1}(\boldsymbol{i})-\bar{I}_{1}\right)\left(I_{2}(\boldsymbol{i})-\bar{I}_{2}\right)\right)^{2}}{\sum_{\boldsymbol{i} \in M}\left(I_{1}(\boldsymbol{i})-\bar{I}_{1}\right)^{2} \sum_{\boldsymbol{i} \in M}\left(I_{2}(\boldsymbol{i})-\bar{I}_{2}\right)^{2}}
$$

is the square of the well-known correlation coefficient [8].

In the case of a linear relationship between the gray-values of the software phantom images, the quantity $D^{\prime}(\boldsymbol{\omega}, \boldsymbol{t})$ should take its global minimum value $(=0)$ and the square of the correlation coefficient its global maximum $(=1)$ if the images are registered. Because there is no linear relationship, the quantity $D^{\prime}(\boldsymbol{\omega}, \boldsymbol{t})$ has a larger value (354) and the square of the correlation coefficient is smaller (0.36). The maximum of the squared correlation coefficient found during registration (0.49) does no more correspond to a proper registration showing that the correlation coefficient is in general not suitable for multi-modality registration.

\subsection{Local Correlation}

To evaluate local correlation, for a pixel $\boldsymbol{i}$ in the reference image a spherical neighborhood $S_{\boldsymbol{i}}=\left\{\boldsymbol{l}=\left(l_{x}, l_{y}, l_{z}\right) \mid\left(i_{x}-l_{x}\right)^{2}+\left(i_{y}-l_{y}\right)^{2}+\left(i_{z}-l_{z}\right)^{2} \leq r^{2}\right\}$ of radius $r$ is defined and the squared correlation coefficient $C_{\boldsymbol{i}}^{2}(\boldsymbol{\omega}, \boldsymbol{t})$ between this neighborhood and the corresponding neighborhood in the other image is evaluated:

$$
C_{\boldsymbol{i}}^{2}(\boldsymbol{\omega}, \boldsymbol{t})=\frac{\left(\sum_{\boldsymbol{l} \in S_{\boldsymbol{i}}}\left(I_{1}(\boldsymbol{l})-\bar{I}_{1}(\boldsymbol{i})\right)\left(I_{2}(\boldsymbol{l})-\bar{I}_{2}(\boldsymbol{i})\right)\right)^{2}}{\sum_{\boldsymbol{l} \in S_{\boldsymbol{i}}}\left(I_{1}(\boldsymbol{l})-\bar{I}_{1}(\boldsymbol{i})\right)^{2} \sum_{\boldsymbol{l} \in S_{\boldsymbol{i}}}\left(I_{2}(\boldsymbol{l})-\bar{I}_{2}(\boldsymbol{i})\right)^{2}}
$$

with $\bar{I}_{1}(\boldsymbol{i})$ and $\bar{I}_{2}(\boldsymbol{i})$ denoting the average gray-value in the neighborhood $S_{\boldsymbol{i}}$ for the reference image and the image to be registered. In the case that one of the images has a constant gray-value within a neighborhood $S_{\boldsymbol{i}}$, the contribution $C_{\boldsymbol{i}}^{2}$ is set to zero to avoid undefined results. The local correlation $\operatorname{LC}^{2}(\boldsymbol{\omega}, \boldsymbol{t})$ is obtained by summing up all contributions within the overlap region:

$$
\operatorname{LC}^{2}(\boldsymbol{\omega}, \boldsymbol{t})=\frac{1}{\# M} \sum_{i \in M} C_{i}^{2}(\boldsymbol{\omega}, \boldsymbol{t}) .
$$

The essential difference between the correlation coefficient and local correlation refers to the relationship between the gray-values of the images to be registered. 
The correlation coefficient presumes a global linear relationship whereas local correlation assumes a linear relationship in a local neighborhood only. This linear relationship can be different in different regions of the image.

If the local correlation is applied to the software phantom, the contributions $C_{\boldsymbol{i}}^{2}(\boldsymbol{\omega}, \boldsymbol{t})$ can be divided into three categories:

1. One of the images has a constant gray-value within the neighborhood. In this case, the contribution $C_{\boldsymbol{i}}^{2}(\boldsymbol{\omega}, \boldsymbol{t})$ is zero and can be neglected.

2. Both images show only two different gray-values within the neighborhood $S_{\boldsymbol{i}}$. In this case, the gray-values can be transformed into each other by a linear relationship. The better the structures within the neighborhood match, the larger becomes the contribution $C_{\boldsymbol{i}}^{2}(\boldsymbol{\omega}, \boldsymbol{t})$, therefore.

3. One of the images has at least two and the other one at least three different gray-values within the neighborhood $S_{\boldsymbol{i}}$. In this case, it is usually not possible to map the gray-values onto each other assuming a linear relationship. Thus, the contribution $C_{\boldsymbol{i}}^{2}(\boldsymbol{\omega}, \boldsymbol{t})$ may not take its maximum value, if the images are registered optimally.

As there are only a few regions in the phantom images with three different structures next to each other, there are only few contributions of the last category. Local correlation is dominated by contributions of the second category, therefore. These contributions belong to neighborhoods with two different gray-values - i.e. a gray-value edge - in each image, and these contributions take their maximum values if respective edges are aligned. This is illustrated in Fig. 1c which shows the spatial distribution of the contributions to the local correlation. Contributions can clearly be found in the area of gray-value edges showing that local correlation essentially quantifies corresponding edges.

From the preceding discussion it is evident that all voxels for which the local gray-value variance is zero can be neglected. Because of noise, there will hardly be voxels in real images for which the local variance vanishes. Nevertheless, regions with almost no structures or gray-value edges contribute only little to the local correlation. All voxels with a local gray-value variance below some threshold can be neglected and only a fraction of the image volume is necessary for registration. This property can be used to accelerate registration.

\section{Experiments and Results}

The validation results refer to three data sets. The first data set is the software phantom of Fig. 1. As both images originate from the same image, they are registered implicitly. The second data set is a set of MR images shown in Fig. 2. They have been acquired simultaneously with a multi-slice, dual contrast turbo spin echo sequence. Thus, the spatial correspondence is not disturbed and the images are registered, as well. The third data set is a clinical one. It consists of an MR and a CT image which are shown in Fig. 3. Information about resolution and the size of the voxel matrix of all images can be found in Tab. 2 .

For the experiments, one of the two software phantom images (Fig. 1b) and 
one of the two dual contrast MR images (Fig. 3b) have been reformatted according to a rigid transformation with translation $(10.3 \mathrm{~mm}, 12.7 \mathrm{~mm},-3.5 \mathrm{~mm})$ and rotation $\left(9.4^{\circ}, 11.1^{\circ},-4.2^{\circ}\right)$. For the CT-MR data set a registration has been carried out. Using these parameters, sets with 64 starting estimates have been generated by adding $\pm 11.42 \mathrm{~mm}$ and $\pm 11.42^{\circ}$ to the translations and rotations. Then, registration has been performed for each starting estimate using the multi-resolution algorithm of Studholme et al. [2]. As similarity measure local correlation $(r=1)$, local correlation $(r=1)$ with $10 \%$ of the image volume and mutual information have been used. Optimization was done using three isotropic resolutions of $4.0 \mathrm{~mm}, 2.8 \mathrm{~mm}$ and $2 \mathrm{~mm}$ and a minimum step size of approximately $0.01 \mathrm{~mm}$ and $0.01^{\circ}$. Finally, the mean rotations and translations as well as the corresponding standard deviations have been computed. For the CTMR data set, 5 (local correlation with $10 \%$ of the image volume) and 11 (mutual information) results have been discarded in this final step, because optimization was obviously trapped in a local optimum. The results of all experiments are included in Tab. 3. Fig. 3c shows an overlay of the CT image edges onto the MR image after registration using local correlation.
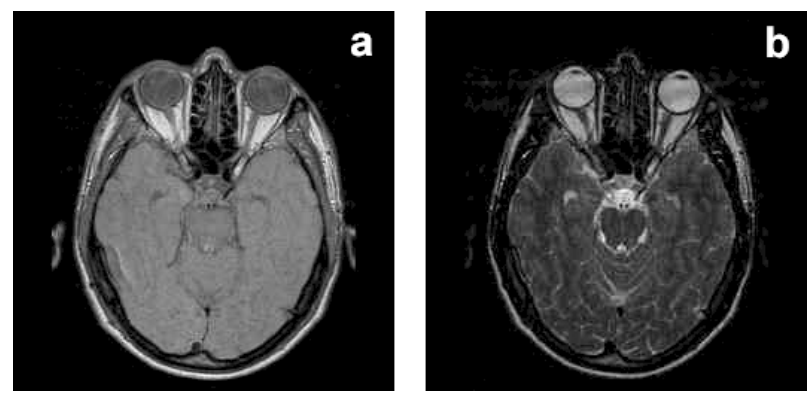

Fig. 2. Simultaneously acquired dual contrast MR images.

Table 2. Dimension and voxel size of the images. For the clinical CT image the slice-to-slice distance is $1 \mathrm{~mm}$

\begin{tabular}{l|c|c|} 
& dimension (in voxels) & voxel size $($ in mm) \\
\hline software phantom & $256 \times 256 \times 144$ & $0.98 \times 0.98 \times 1.2$ \\
\hline dual contrast MR images & $256 \times 256 \times 64$ & $0.90 \times 0.90 \times 3.0$ \\
\hline clinical CT image & $512 \times 512 \times 87$ & $0.41 \times 0.41 \times 3.0$ \\
clinical MR image & $256 \times 256 \times 128$ & $0.78 \times 0.78 \times 1.5$ \\
\hline
\end{tabular}

\section{Discussion}

The results for the software phantom and the dual contrast MR images show that with local correlation as well as with mutual information the registration result is almost independent of the starting estimate and that the "ground-truth" 
registration is recovered with high accuracy. Though the highest resolution used during optimization was $2 \mathrm{~mm}$, the accuracy of the translation and rotation parameters is $0.03 \mathrm{~mm}$ and $0.01^{\circ}$, respectively. If only $10 \%$ of the image volume is used for registration with local correlation, the results are slightly degraded. In the case of the dual contrast MR images, the inaccuracies increase to $0.13 \mathrm{~mm}$ and $0.05^{\circ}$. Nevertheless, the translation inaccuracy remains to be well below the voxel size used for registration and the rotation inaccuracy is very small.

Table 3. Registration results. Since optimization was obviously trapped in a local optimum, $5\left(\mathrm{LC}_{10 \%}^{2}\right)$ and $11(\mathrm{MI})$ registration results have been discarded for the clinical CT-MR data set.

\begin{tabular}{l|l|l|l|l|l|l|} 
& \multicolumn{5}{|c|}{ translations (in mm) } & \multicolumn{3}{c|}{ rotations ( ${ }^{\circ}{ }^{\circ}$} \\
\hline & \multicolumn{5}{|c|}{ Software Phantom } \\
\hline $\mathrm{LC}^{2}$ & $10.30 \pm 0.01$ & $12.69 \pm 0.01$ & $-3.49 \pm 0.01$ & $9.41 \pm 0.01$ & $11.12 \pm 0.02$ & $-4.19 \pm 0.01$ \\
\hline $\mathrm{LC}_{10 \%}^{2}$ & $10.31 \pm 0.01$ & $12.67 \pm 0.01$ & $-3.50 \pm 0.02$ & $9.40 \pm 0.01$ & $11.11 \pm 0.03$ & $-4.20 \pm 0.03$ \\
\hline $\mathrm{MI}$ & $10.30 \pm 0.00$ & $12.70 \pm 0.00$ & $-3.50 \pm 0.00$ & $9.40 \pm 0.01$ & $11.11 \pm 0.00$ & $-4.20 \pm 0.00$ \\
\hline & \multicolumn{5}{|c|}{ Dual Contrast MR images } \\
\hline $\mathrm{LC}^{2}$ & $10.30 \pm 0.01$ & $12.71 \pm 0.01$ & $-3.51 \pm 0.01$ & $9.39 \pm 0.01$ & $11.11 \pm 0.01$ & $-4.20 \pm 0.01$ \\
\hline $\mathrm{LC}_{10 \%}^{2}$ & $10.30 \pm 0.01$ & $12.83 \pm 0.01$ & $-3.47 \pm 0.01$ & $9.35 \pm 0.01$ & $11.11 \pm 0.01$ & $-4.21 \pm 0.01$ \\
\hline $\mathrm{MI}$ & $10.29 \pm 0.01$ & $12.71 \pm 0.01$ & $-3.47 \pm 0.02$ & $9.41 \pm 0.03$ & $11.11 \pm 0.03$ & $-4.20 \pm 0.02$ \\
\hline & \multicolumn{6}{|c|}{ Clinical CT-MR data set } \\
\hline $\mathrm{LC}^{2}$ & $-3.49 \pm 0.02$ & $-16.09 \pm 0.02$ & $-31.00 \pm 0.01$ & $6.92 \pm 0.02$ & $-2.21 \pm 0.02$ & $-5.13 \pm 0.01$ \\
\hline $\mathrm{LC}_{10 \%}^{2}$ & $-3.46 \pm 0.01$ & $-16.06 \pm 0.02$ & $-30.69 \pm 0.01$ & $6.81 \pm 0.01$ & $-2.44 \pm 0.01$ & $-5.11 \pm 0.01$ \\
\hline $\mathrm{MI}$ & $-3.33 \pm 0.07$ & $-16.06 \pm 0.11$ & $-30.74 \pm 0.12$ & $6.79 \pm 0.13$ & $-2.15 \pm 0.13$ & $-5.00 \pm 0.09$ \\
\hline
\end{tabular}

In the case of the clinical data set, registration was only successful for all starting estimates when using local correlation and the entire image volume.
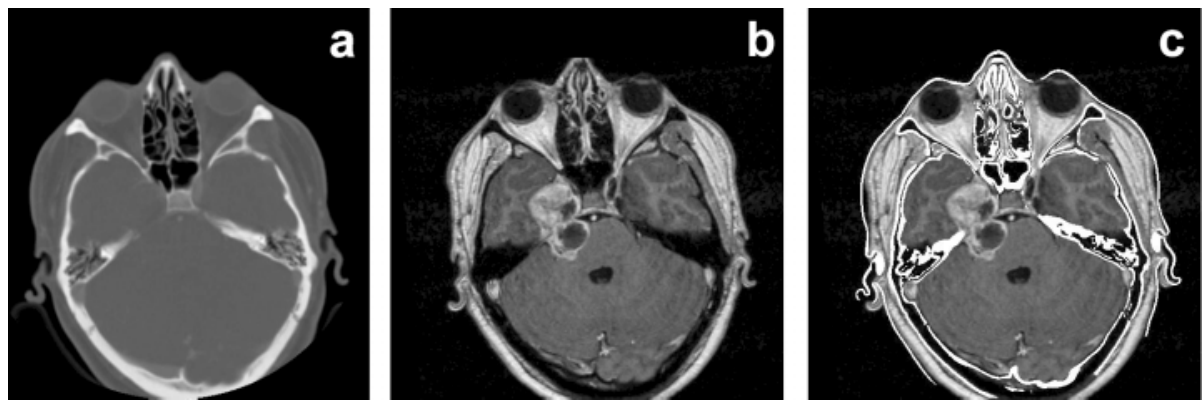

Fig. 3. Clinical data set. CT image (a), MR image (b) and overlay of the CT image edges onto the MR image after registration with local correlation (c). 
When using only $10 \%$ of the image volume or mutual information, the capture range was slightly smaller and registration was trapped 5, respectively 11 times in local optima. The variations of the registration result for different starting estimates are larger for mutual information than for local correlation, though these variations are small and the registration result is nearly independent of the starting estimate. The deviations between the results for mutual information and local correlation have a magnitude of up to $0.26 \mathrm{~mm}$ for the translations and $0.13^{\circ}$ for the rotations. For local correlation using $10 \%$ of the image volume, the deviation for the rotations increases to $0.29^{\circ}$. Because mutual information has been used successfully for CT-MR registration, the small deviations are a strong indication that local correlation leads also to a proper registration. The good agreement of corresponding structures in the CT and MR image after registration (see Fig. 3c) confirms that local correlation is suitable for CT-MR registration.

\section{Conclusions}

Registration of CT and MR images using local correlation has been investigated. The differences between correlation and local correlation have been discussed, and it has been shown that local correlation quantifies corresponding edges in the images to be registered. There is, therefore, some analogy to the approach of Maintz et al. [3] where edges or ridges are extracted and registration is carried out on the basis of the feature images. An important difference is that registration with local correlation requires no explicit feature extraction. It can be applied directly to the gray-value images without sophisticated pre-processing. Since local correlation quantifies corresponding edges, image regions with almost no structures do not significantly contribute to the measure. Such image regions can easily be identified using a threshold for the local gray-value variance and can be excluded during registration. This technique allows to compensate a disadvantage related to the numerical evaluation of local correlation which is rather time consuming, because a neighborhood must be considered for each voxel.

Application to a software phantom and two MR images with different contrast led to accurate registration results being as good as the results obtained with mutual information. The good agreement between the registration results computed with local correlation and mutual information for a clinical CT-MR data set gave further evidence for the suitability of local correlation for multi-modality registration. The experiments showed also that registration based on local correlation can be done using only $10 \%$ of the image volume.

\section{Acknowledgments}

We would like to thank C. Studholme, D.L.G. Hill and D.J. Hawkes (UMDS, Guy's and St. Thomas' Hospitals, London) for the multi-resolution algorithm and our colleagues at the MR department (Philips Research Hamburg) for providing the dual contrast images. 


\section{References}

1. Maintz, J. B. A., Viergever, M. A.: A survey of medical image registration. Med. Image Anal. 2 (1998) 1-36 656

2. Studholme, C., Hill, D.L.G., Hawkes, D.J.: Automated 3-D registration of MR and CT images of the head. Med. Image Anal. 1 (1996) 163-175 656, 657, 661

3. Maintz, J. B. A., van den Elsen, P. A., Viergever, M.A.: Comparison of edge-based and ridge-based registration of $\mathrm{CT}$ and MR brain images. Med. Image Anal. 1 (1996) 151-161 656, 663

4. Wells III, W.M., Viola, P., Atsumi, H., Nakajima, S., Kikinis, R.: Multi-modal volume registration by maximization of mutual information. Med. Image Anal. 1 (1996) 35-51. 657

5. Maes, F., Collignon, A., Vandermeulen, D., Marchal, G., Suetens, P.: Multimodality image registration by maximization of mutual information. IEEE Trans. Med. Imag. 16 (1997) 187-198. 657

6. Rösch, P., Blaffert, T., Weese, J.: Multi-modality registration using local correlation, accepted for CARS'99 657

7. Vincent, L., Soille, P.: Watersheds in digital spaces: an efficient algorithm based on immersion simulations. IEEE Trans. Pat. Anal. Mach. Int. 13 (1991) 583-598. 657

8. Buzug, T.M., Weese, J.: Voxel-based similarity measures for medical image registration in radiological diagnosis and image guided surgery. J. Comput. Inf. Tech. 6 (1998) 165-179. 659 